\title{
2.45 GHz Class E Power Amplifier for a Transmitter Combining LINC and EER
}

\author{
M. Dirix, O. Koch \\ A 10 W class-E RF power amplifier (PA) is designed and fabricated using a Cree GaN HEMT. The proposed PA uses an innovative input \\ circuit to optimize band with. At $2.45 \mathrm{GHz}$ the PA achieves a PAE of $60 \%$ at an outputpower of $40 \mathrm{dBm}$. The resulting amplifier is simu- \\ lated and constructed using a transmissionline topology. Two of these amplifiers are fabricated on a single board for outphasing application. \\ Their suitability for outphasing application and supply modulation is investigated.
}

Keywords: Class E, power amplifier, CLIER.

\section{Introduction}

Efficiency is a more and more critical issue when designing transmitters, both to save energy costs at the base station and to reduce the power consumption of mobile terminals to increase battery life. Efficiency boosting techniques tend to rely on switch-mode amplifiers (SMA). Conventional amplifier classes like $\mathrm{A}, \mathrm{AB}$, which offer high linearity at the cost of efficiency are avoided. The non-linearities caused by these amplifiers are compensated using envelope elimination and restoration (EER)[2] or vector addition (LINC) [1] techniques or both (CLIER) [5].

For implementation of a CLIER amplifier architecture a Class E amplifier is well suited both for the LINC and for the EER part. LINC consists of two non-linear amplifiers. The input signal, containing both amplitude $a(t)$ and phase $\varphi(t)$ is transformed into two solely phase modulated signals with constant envelopes. Both non-linear amplified signals are vector combined at the output, resulting in an amplified replica of the original time varying envelope signal. Additionally, the supply of the amplifiers is modulated with a slowly varying signal. This reduces the dynamics of the LINC signal and thereby increases the efficiency of the entire system. This paper focuses on the implementation of class $\mathrm{E}$ amplifiers.

\section{Theory}

For lossless operation of the Class E amplifier there are two criteria that have to be met: First, zero voltage across the transistor terminals when the switch closes [4]

$$
u_{s}\left(\omega t=\omega t_{c}\right)=0 \text {. }
$$

And, second, no voltage building across the transistor terminals while the switch is closed

$$
\frac{\mathrm{d} u_{s}}{\mathrm{~d} t}\left(\omega t=\omega t_{c}\right)=0 \text {. }
$$

To obtain this non overlapping between voltage and current a complex load impedance is imposed on the switch output terminals, which results in a phase shift between current and voltage of the output signal.

Fig. 1 shows a typical Class E circuit. The transistor is ideally replaced with a switch. The load network consists of a parallel capacitor $\mathrm{C}$ and an inductance $\mathrm{L}$ in series with a tuned output network $L_{0} C_{0}$ and load resistance $\mathrm{R}$. When using a real transistor the capacitor $\mathrm{C}$ can comprise the intrin-

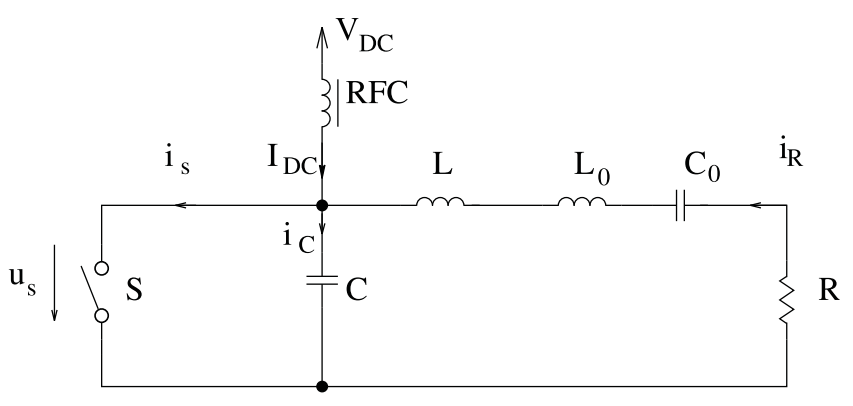

Fig. 1: Class E circuit with lumped elements

sic transistor capacitance $C_{d s}$ and an external capacitance. An RF choke at the supply voltage enforces a DC supply current.

The output tuning network $L_{0} C_{0}$ forces the current through the load resistance $\mathrm{R}$ to be a sinusoidal function of $\omega t$ angular time and phase shift $\varphi$. The current can be described as

$$
i_{R}(\omega t)=I_{R} \sin (\omega t+\varphi) .
$$

Because the RF choke enforces a DC current $I_{D C}$, the difference between output- and DC current flows into the switch-capacitance network. Initially, when the switch $\mathrm{S}$ is closed, the switch current is zero $i_{s}(\omega t=0)=0$. With (3), the DC current can then be described as

$$
I_{D C}=-I_{R} \sin (\varphi) \text {. }
$$

While the switch $\mathrm{S}$ is closed the capacitor $\mathrm{C}$ has zero voltage across and consequently all current starts flowing through $\mathrm{S}$ for $\omega t>0$. The current flowing the switch $\mathrm{S} i_{s}(\omega t)$ can be described as

$i_{s}(\omega t)=I_{D C}+I_{R} \sin (\omega t+\varphi)=I_{R}[\sin (\omega t+\varphi)-\sin (\varphi)]$.

At the time $\omega t=\omega t_{0}=\pi+k 2 \pi$ the switch $\mathrm{S}$ opens and the difference current $i_{c}\left(\omega t-\omega t_{0}\right)=i_{s}\left(\omega t+\omega t_{0}\right)$ flows into capacitor $\mathrm{C}$ which starts charging. The build up of the voltage $u_{s}(\omega t)$ across the switch $\mathrm{S}$ is determined by the charging of capacitor $\mathrm{C}$, which can be described as

$$
u_{s}(\omega t)=\frac{1}{\omega C} \int_{\omega t_{0}}^{\omega t} i_{c}(\omega t) \mathrm{d} \omega t .
$$


With (5) it follows that

$u_{s}(\omega t)=-\frac{I_{R}}{\omega C}[\cos (\omega t+\varphi)+\cos \varphi+(\omega t-\pi) \sin \varphi]$.

With the first criteria for Class E operation Eq. (1) and Eq. (7) it follows that for optimal operation the phase angle $\varphi$ can be defined as

$$
\varphi=\tan ^{-1}\left(-\frac{2}{\pi}\right)=-32.482 \mathrm{deg} .
$$

With these equations the resulting normalized voltage can easily be obtained. The normalized voltage and current transients are shown in Fig. 2. As can be seen, there is no overlap between switch current and voltage waveforms, resulting in $100 \%$ switch efficiency.

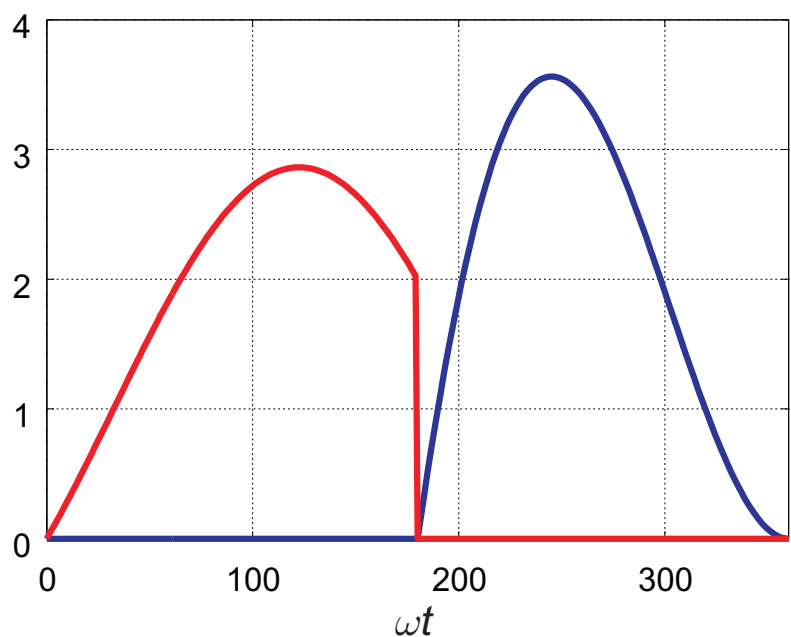

Fig. 2: Switch current (light) and voltage (dark) transient

A common design approach for high frequency Class E PAs is to design a lumped element amplifier [4] and then replace each component with an equivalent transmission line.

This however makes the design and optimization both tedious and time-consuming because only the slightest change in a transmission line parameter will change the load network radically, losing Class E operating conditions.

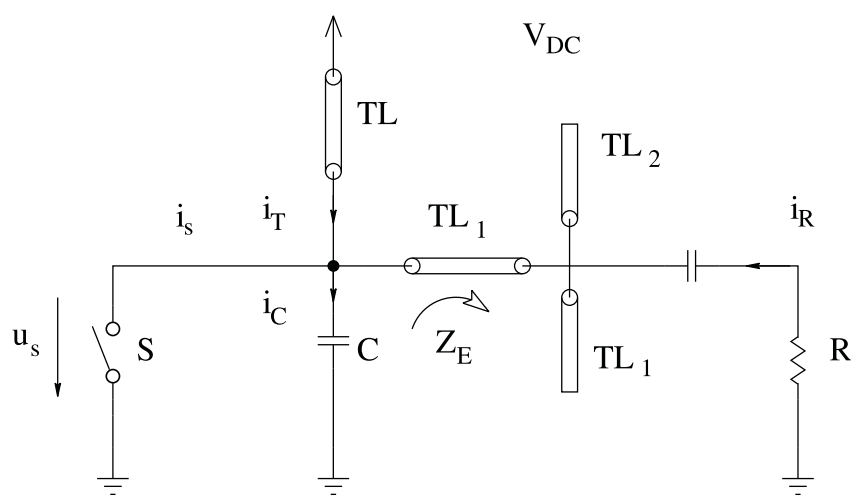

Fig. 3: Class E circuit with transmission lines
A better approach is found in [3]. Consider Fig. 3. Class E operation in this circuit depends on the load network providing a phase angle $\varphi=49.05 \mathrm{deg}$. This complex output impedance, using $\tan \phi=X / R$, can be written as

$$
Z_{E}=\left\{\begin{array}{lll}
R_{E}(1+j 1.152) & \text { at } & f_{0} \\
\infty & \text { at } & n f_{0}, n>1 .
\end{array}\right.
$$

The optimum value for the load resistance $R_{E}$ at the fundamental frequency $f_{0}$ is given by

$$
R_{E}=\frac{1}{34.225 f_{0} C_{s}} \text {. }
$$

By choosing a moderate to high impedance for transmission lines $Z_{T L 1}$ and $Z_{T L 2}$ and defined output load impedance $R_{L}$, circuit parameters can be further obtained by keeping the absolute value of the reflection parameter on both sides of $T L 1$ equal $\left|\Gamma_{E}\right|=\left|\Gamma_{G}\right|$. The total admittance combining the load admittance $G_{L}$ and transmission lines TL2 and TL3 can be written as

$$
Y_{G}=G_{L}+j B
$$

where $B$ is

$$
B=\sqrt{\frac{\left|\Gamma_{E}\right|^{2} \cdot\left(Y_{T L 1}+G_{L}\right)^{2}-\left(Y_{T L 1}-G_{L}\right)^{2}}{1-\left|\Gamma_{E}\right|^{2}}}
$$

Because the load resistance is real, the imaginary part of $Y_{G}$ has to comprise both transmission lines TL2 and TL3. With the already chosen parameter $Z_{T L 2}, Z_{T L 3}$ can be obtained with

$$
Z_{T L 3}=\frac{1}{B-\frac{1}{Z_{T L 2}}} \cdot \tan (30 \mathrm{deg}) .
$$

With $\Gamma_{E}$ being the reflection factor at the transistor output terminals

$$
\Gamma_{E}=\frac{Z_{E}-Z_{T L 1}}{Z_{E}+Z_{T L 1}}
$$

and $\Gamma_{G}$ the reflection factor at the end of transmission line $T L_{1}$

$$
\Gamma_{G}=\frac{\frac{1}{Y_{G}}-Z_{T L 1}}{\frac{1}{Y_{G}}+Z_{T L 1}}
$$

the electrical length of $Z_{T L 1}$ can be calculated with

$$
\frac{l_{T L 1}}{\lambda}=\frac{\ln \left(\frac{\Gamma_{E}}{\Gamma_{G}}\right)}{-j 4 \pi} .
$$

Because this implementation only provides a high impedance at the second and third harmonics across the transistor output terminals, output current and voltage waveforms are not completely separated resulting in less than $100 \%$ maximum efficiency. Hence the transmissionline circuit should only be used for applications where it is not possible to use 
lumped elements, or the insertion losses are greater than the efficiency restraints due to harmonic termination problems.

\subsection{Input network}

To meet the demands that Class E imposes on the used transistor for this project a GaAS HEMT transistor of type CREE CGH4010F is chosen. This transistor allows for a maximum collector voltage of $120 \mathrm{~V}$. The input impedance for this transistor is $4-j 4 \Omega$ at $2.4 \mathrm{GHz}$ which needs to be adapted to the $50 \Omega$ output of the pre-amplifier. While this can be easily be achieved using a $\lambda / 4$ transmission line limiting bandwidth, a binomial input filter is chosen. The main advantage of using a binomial matching transformer is that the passband response is maximally flat near the design frequency. The order $N$ also determines the number of sections in the transformer [7].

\subsection{Simulation and Measurement Results}

In this section, the Class $\mathrm{E}$ amplifier structure is modeled and simulated in the Agilent Advanced Design System (ADS). Then simulation results are compared to measurement of the implemented amplifier. The Cree CGH4010F GaN HEMT has an output capacitance $C_{G S}$ of typically $1.3 \mathrm{pF}$ [6]. Using Eqs. (10) and (9) the required load impedance $Z_{E}$ can be calculated. Further following the advice of [3] a medium to high characteristic impedance has been chosen for $T L_{1}=75 \Omega$ and $T L_{2}=50 \Omega$. Further parameters can easily be attained using equations presented in Section 2.

First, the circuit output network is simulated using two real ports, to review the location of the second and third harmonic in a Smith chart. As already mentioned, the output network should impose a $40.5 \mathrm{deg}$ phase shift on the transistor output

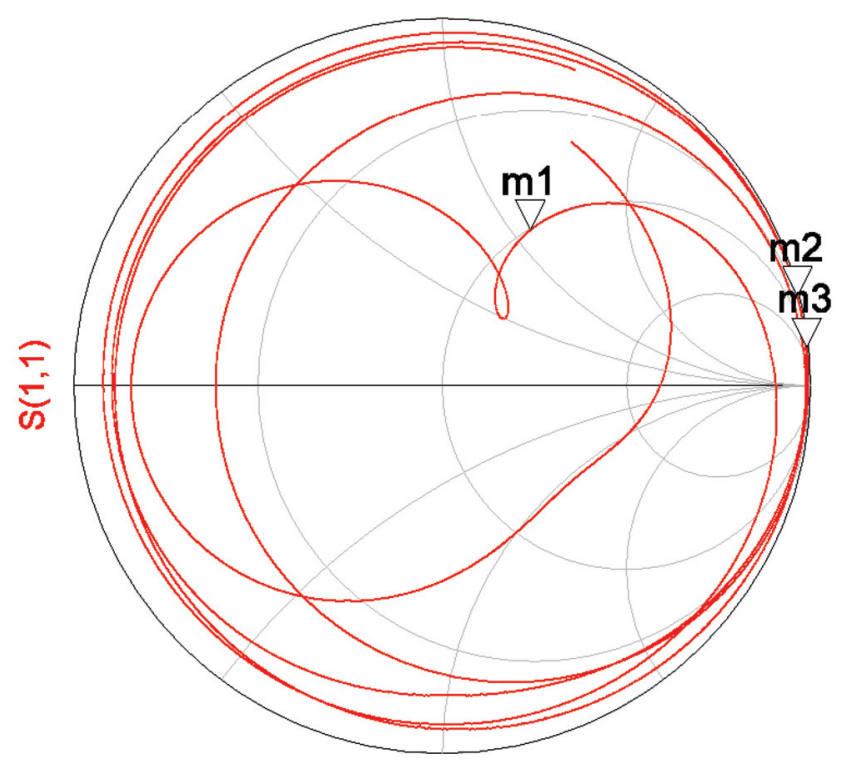

Fig. 4: Smithchart of the output load network

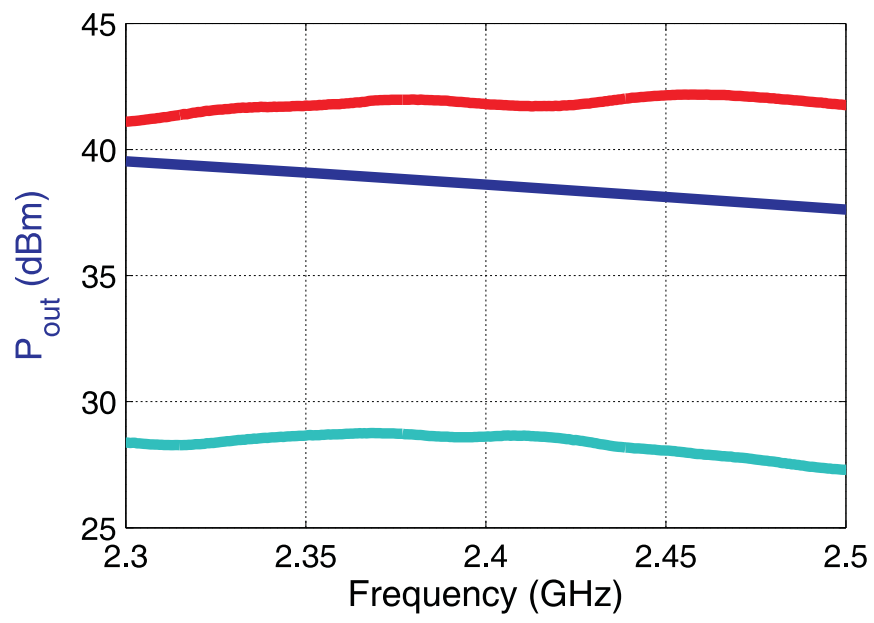

Fig. 5: Frequency response of the measured and simulated amplifier, and pre-amplifier using a $20 \mathrm{~V}$ DC supply voltage

terminals for the target frequency and impose a high impedance at the second and third harmonic. Fig. 4 shows the frequency response of the output network as seen by the transistor output terminals.

Fig. 5 shows the frequency response of the amplifier. In red the measurement, and in blue the simulated values. Remarkable for this amplifier is that the measurement results are well above the simulation results. In addition, the real amplifier performs best with $28 \mathrm{dBm}$ input power, while the simulation is carried out with $30 \mathrm{dBm}$ input power. These differences can be explained given that the simulation model of the transistor is optimized for Class $\mathrm{AB}$ operation. The output frequency response of the pre-amplifier is shown in turquoise.

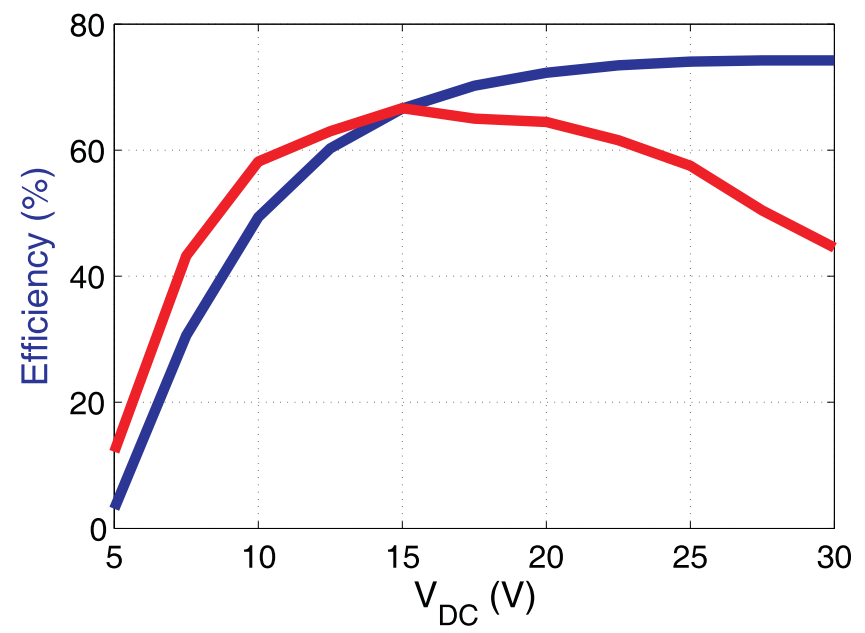

Fig. 6: Power added efficiency

Fig. 6 shows the resulting amplifier efficiency.

Fig. 7 shows that the two amplifiers, Yellow and Blue, are put together on a single circuit board for implementation in 
the CLIER system. In the following figures the two amplifiers can be compared.

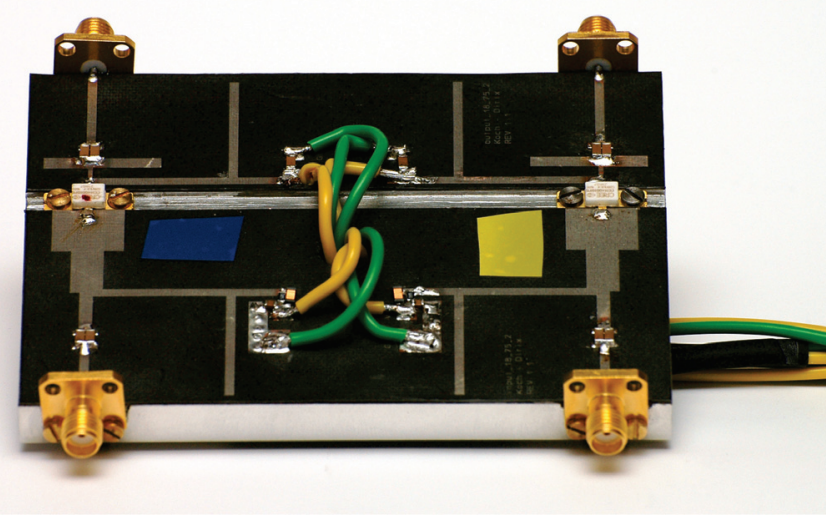

Fig. 7: The two Linc Class E amplifiers on a circuitboard

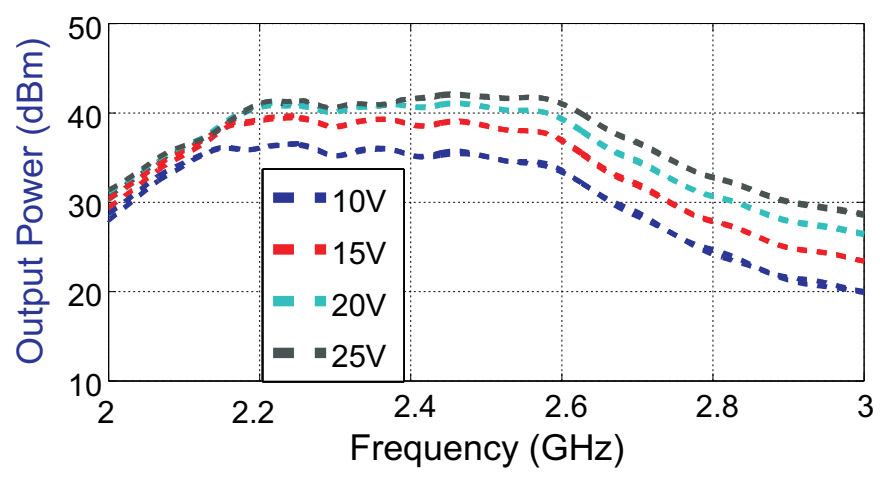

Fig. 8: Frequency response of the blue and the yellow amplifier with 10, 15, 20 and $25 \mathrm{~V}$ supply voltage, DC supply

Figs. 8 and 9 show that the differences between the two amplifiers are small. This makes them ideally suited for further implementation in the LINC part of the CLIER amplifier architecture, as a LINC transmitter requirers a small phase and amplitude imbalance between the two paths. Figure 10 depicts the linearity between the DC supply voltage and the output voltage. As can be seen good linearity is delivered up to $20 \mathrm{~V}$ DC. The non-linearity in the higher voltage range must be compensated using pre-distorting or with the LINC part of the architecture.

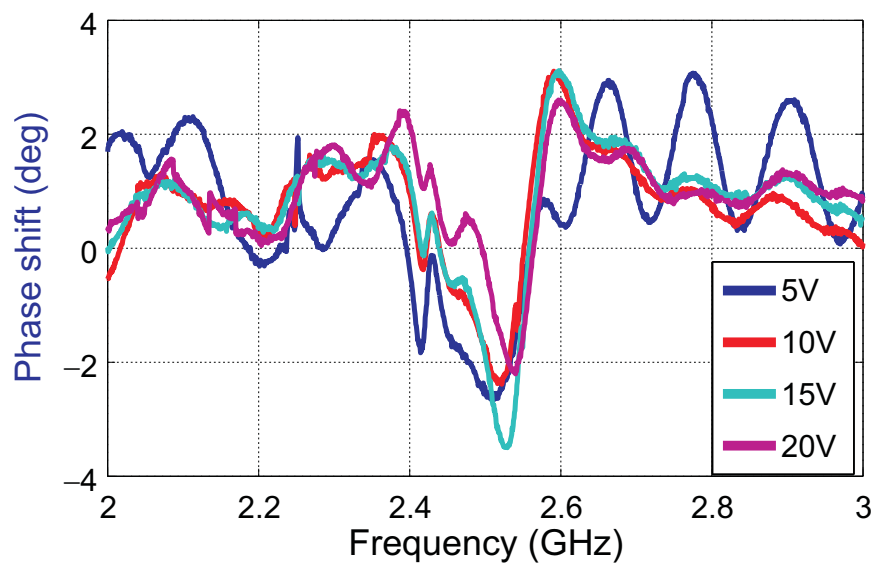

Fig. 9: Phase difference of the two ouput signals B21-Y21

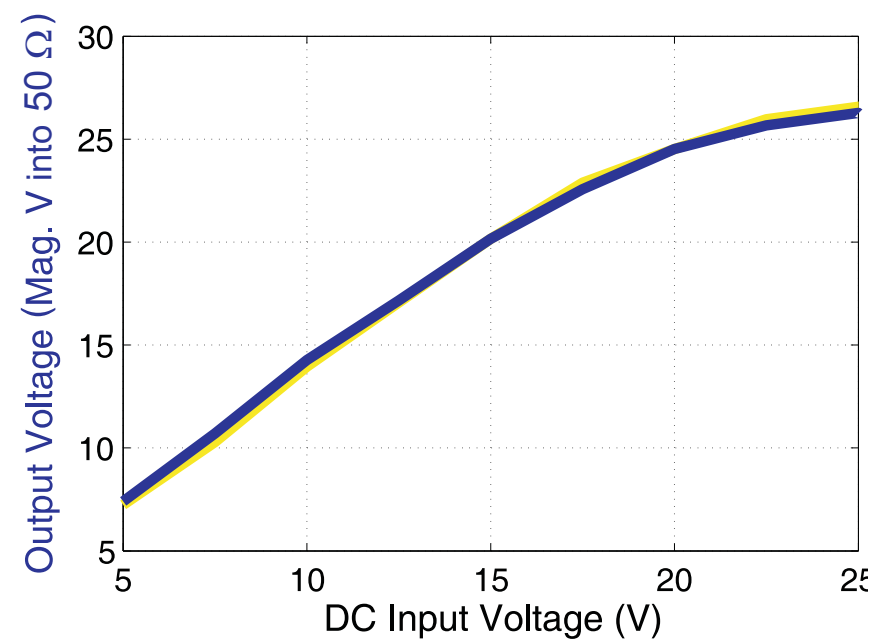

Fig. 10: Comparison of the output voltage of the yellow (light) and the blue (dark) amplifier

\section{Conclusion}

A Class E Power Amplifier has been presented and its efficiency simulated and measured. Its suitability for a CLIER transmitter has been demonstrated.

\section{References}

[1] Cox, D. C.: Linear Amplification with Nonlinear Components. IEEE-TC, Vol. 41 (1974), No. 4, p. 1942-1945.

[2] Kahn, L. R.: Signal-Sideband Transmission by Envelope Elimination and Restoration. Radioengineering, Vol. 40 (1952), p. 803-806.

[3] Negra, R., Fadhel, M., Ghannouchi, M., Bachtold, W.: Study and Design Optimization of Multiharmonic Transmission-Line Load Networks for Class-E and Class-FK-Band MMIC Power Amplifiers. IEEE Transactions on Microwave Theory and Techniques, 207, Vol. 55, No. 6, p. 1390-1394.

[4] Raab, F. H.: Idealized Operation of the Class E Tuned Power Amplifier. IEEE Transactions on Circuits and Systems, Vol. 24 (1977), No. 12, p. 725-735.

[5] Rembold, B., Koch, O.: Increasing Power Amplifier Efficiency by Combining LINC and EER, Proceedings of the $12^{\text {th }}$ International Student Conference on Electrical Engineering POSTER 2008.ue (Czech Republic), 2008.

[6] Cree Inc.: CGH40010 Datasheet 2007, Rev 1.5 Preliminary

[7] Pozar, D. M.: Microwave Engineering, John Wiley \& Sons, Inc., 2005.

Marc Dirix

e-mail: marc@ihf.rwth-aachen.de

Olivier Koch

e-mail:koch@ihf.rwth-aachen.de

Institute of High Frequency Technology

RWTH Aachen University

Melatener Strasse 25

52074 Aachen, Germany 